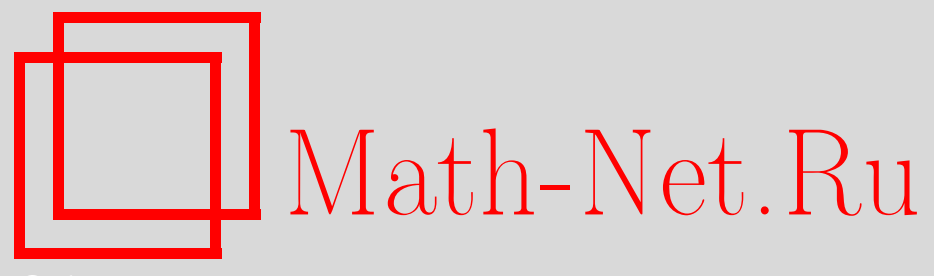

А. М. Магомедов, Т. А. Магомедов, О приложении алгоритнма вычисления подграфа максимальной плотности к задаче оптимизации расписания, Матем. заметки, 2013, том 93, выпуск 2, 313-315

DOI: https://doi.org/10.4213/mzm8770

Использование Общероссийского математического портала Math-Net.Ru подразумевает, что вы прочитали и согласны с пользовательским соглашением http://www . mathnet.ru/rus/agreement

Параметры загрузки:

IP: 54.224 .135 .184

26 апреля 2023 г., 09:53:21

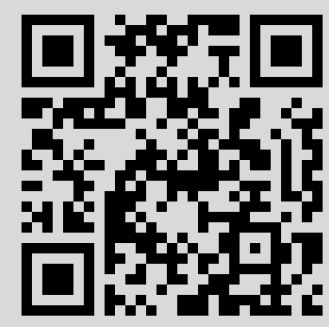




\section{О приложении алгоритма вычисления подграфа максимальной плотности к задаче оптимизации расписания}

\section{А. М. Магомедов, Т. А. Магомедов}

1. Задача вычисления плотности графа. Под плотностью графа понимают отношение числа ребер к числу вершин в графе. Для неориентированного графа $G=(V, E)$, $|V|=n,|E|=m$, в [1] построен быстрый алгоритм вычисления подграфа графа $G$, обладающего максимальной плотностью (в дальнейшем тексте $-\operatorname{density}(G))$. Если $M(v, e)-$ время, требуемое для нахождения пропускной способности минимального разреза в сети с $v$ вершинами и е ребрами, то алгоритм [1] вычисляет значение $\operatorname{density}(G)$ за время

$$
O(M(n, n+m) \log n) .
$$

В данном сообщении предлагается подход к вычислению $\operatorname{density}(G)$, отличный от подхода, принятого в [1], и рассмотрено его применение к проверке условий существования непрерывного расписания обслуживания.

2. Сведение к вычислению паросочетаний в двудольных графах. Для заданного графа $G=(V, E), V=\left\{v_{1}, \ldots, v_{n}\right\}, E=\left\{e_{1}, \ldots, e_{l}\right\}$, построим двудольную интерпретацию $G_{1}=\left(X_{1}, Y_{1}, E_{1}\right): X_{1}=\left\{x_{1}, \ldots, x_{l}\right\}, Y_{1}=\left\{y_{1}, \ldots, y_{n}\right\}, d_{G_{1}} x_{i}=2$ для всех $x_{i} \in X_{1}$; $\left(x_{i}, y_{j}\right) \in E_{1}$ в том и только в том случае, если ребро $e_{i}$ и вершина $v_{j}$ инцидентны в графе $G$.

Пусть $\Gamma_{1}(S)$ обозначает множество вершин, смежных в графе $G_{1}$ с вершинами множества $S \subseteq X_{1}$; отношение $|S| /\left|\Gamma_{1}(S)\right|$ есть плотность подграфа, порожденного в графе $G$ подмножеством, образованном ребрами, соответствующими вершинам множества $S$;

$$
\operatorname{density}(G)=\max _{S \subseteq X_{1}}\left\{\frac{|S|}{\left|\Gamma_{1}(S)\right|}\right\} .
$$

Для целого положительного $p$ определим граф $G^{(p)}=\left(X^{(p)}, Y^{(p)}, E^{(p)}\right)$ следующим образом: положим $X^{(p)}=X_{1}$ и для каждой вершины $y \in Y_{1}$ и всех инцидентных ей в графе $G_{1}$ ребер $\left(x_{1}, y\right), \ldots,\left(x_{k}, y\right)$ выполним замену на конструкцию из $p$ новых вершин $y_{1}, \ldots, y_{p} \in Y^{(p)}$ и $p k$ новых ребер $\left(x_{j}, y_{i}\right) \in E^{(p)} ; j=1, \ldots, k, i=1, \ldots, p$. Множество вершин, смежных в графе $G^{(p)}$ с вершинами множества $S \subseteq X_{p}$, обозначим $\Gamma^{(p)}(S)$. Так как $\left|\Gamma^{(p)}(S)\right|=p\left|\Gamma_{1}(S)\right|$, условия

$$
\frac{|S|}{\left|\Gamma_{1}(S)\right|} \leqslant p \quad \forall S \subseteq X_{1}
$$

равносильны условиям

$$
\frac{|S|}{\left|\Gamma^{(p)}(S)\right|} \leqslant 1 \quad \forall S \subseteq X^{(p)},
$$

которые согласно теореме Холла $[2 ; 8.23]$ суть условия существования в графе $G^{(p)}=\left(X^{(p)}\right.$, $Y^{(p)}, E^{(p)}$ ) полного паросочетания множества $X^{(p)}$ в множество $Y^{(p)}$. Таким образом, вычисление $\max _{S \subseteq X_{1}}\left\{|S| /\left|\Gamma_{1}(S)\right|\right\}$ сводится к нахождению в множестве $\{1, \ldots, n\}$ наибольшего элемента $p$, для которого в соответствующем графе $G^{(p)}=\left(X^{(p)}, Y^{(p)}, E^{(p)}\right)$ существует полное паросочетание множества $X^{(p)}$ в множество $Y^{(p)}$. Применение дихотомии приведет к появлению в оценке сомножителя $\log n$, аналогичного сомножителю в оценке (1); напомним, что вычисление максимального паросочетания в двудольном графе из $k$ вершин может быть выполнено за время $O\left(k^{5 / 2}\right)$ [3].

DOI: $10.4213 / \operatorname{mzm} 8770$ 
3. Приложение. Пусть исходные данные к расписанию обслуживания $n$ требований $l$ приборами заданы в виде графа $G=(V, E), V=\left\{v_{1}, \ldots, v_{n}\right\}, E=\left\{e_{1}, \ldots, e_{l}\right\}$ : если $e_{i}=\left(v_{k}, v_{j}\right)$, то набор из двух чисел $k$ и $j$ обозначим $\omega_{i} ; i$-й прибор должен выполнить одну операцию с каждым из требований (необязательно различных) из набора $\omega_{i}$; семейство наборов $\omega_{i}, i=1, \ldots, l$, обозначим $\Omega$. Предполагается, что длительность каждой операции равна единице, порядок выполнения операций произволен, условия предшествования отсутствуют, запрещается одновременное обслуживание одним прибором более одного требования и одного требования более чем одним прибором.

Задача о существовании непрерывного расписания, соответствующего исходным данным, формулируется следующим образом (через $q$ обозначена максимальная степень вершины в графе $G)$ : существует ли матрица $T$ из $l$ строк и $q$ столбиов и с элементами из множества $\{0,1, \ldots, n\}$ такал, что ненулевые элементы в каждом столбце матрицы попарно различны, в каждой $i$-й строке ненулевые элементы образуют набор $\omega_{i}$ и располагаются в соседних ячейках строки?

Интерпретация: если $T_{i, j}=k$, то в $j$-ю единицу времени прибор $i$

a) выполяет операцию над требованием $k$, если $k>0$;

б) не обслуживает никакого требования, если $k=0$.

При $l \geqslant 2$ и отсутствии ограничений на количество требований, предписанных отдельным приборам, в [4] доказана $N P$-полнота задачи о непрерывном расписании.

Равносильная формулировка: требуется проверить возможность раскраски $q$ цветами ребер двудольной интерпретации $G_{1}$ графа $G$ так, чтобы

а) для каждой вершины $x_{i} \in X$ цвета инцидентных ребер отличались точно на 1 ;

б) для каждой вершины $y_{j} \in Y$ все инцидентные ребра имели разные цвета.

Вопросы $N P$-трудности проблемы в общем виде рассмотрены в [5].

Для четных $q$ непрерывное расписание всегда существует [6]. Мы рассматриваем случай, когда $q$ нечетно, $q=2 p+1$. Несмотря на простоту формулировки, для этого случая неизвестно даже, разрешима ли задача за полиномиальное время. В [7] приведен развернутый обзор по данной проблематике.

Пусть искомое непрерывное расписание $T$ существует. Обозначим: $N\left(\Omega^{\prime}\right)$ - количество различных чисел в наборах $\omega_{i}$ подсемейства $\Omega^{\prime} \subseteq \Omega ; \Omega_{i}^{\prime}$ - подсемейство, образованное в $\Omega^{\prime}$ наборами, один из элементов каждого из которых расположен в столбце с номером $2 i$ расписания $T, i=1, \ldots, p$. Легко видеть, что

$$
\left|\Omega^{\prime}\right| \leqslant p \cdot \max _{i=1, \ldots, p}\left|\Omega_{i}^{\prime}\right| \leqslant p \cdot N\left(\Omega^{\prime}\right) .
$$

Итак, для существования непрерывного расписания необходимо выполнение условий

$$
\frac{\left|\Omega^{\prime}\right|}{N\left(\Omega^{\prime}\right)} \leqslant p \quad \forall \Omega^{\prime} \subseteq \Omega
$$

Эти условия равносильны условиям (3). Таким образом, мы доказали, что их проверка сводится к вычислению $\operatorname{density}(G)$.

В [8] доказано, что при $p=1,2$ эти условия являются и достаточными для существования непрерывного расписания.

ГИПотЕЗА. Условия (4) являются необходимыми и достаточными для существования непрерывного расписания в общем случае. 


\section{СПИСОК ЦИТИРОВАННОЙ ЛИТЕРАТУРЫ}

[1] A. V. Goldberg, Finding a Maximum Density Subgraph, Technical Report No. UCB/ CSD-84-171, University of California, Berkeley, CA, 1984. [2] М. Свами, К. Тхуласираман, Графыь, сети и алгоритмы, Мир, М., 1984. [3] J. E. Hopcroft, R. M. Karp, SIAM J. Comput., 2 (1973), 225-231. [4] В. С. Танаев, Ю.Н. Сотсков, В. А. Струсевич, Теория расписаний. Многостадийные системы, Экономико-математическая библиотека, Наука, М., 1989. [5] В. Г. Визинг, Дискретн. анализ и исслед. опер., сер. 1, 12:3 (2005), 48-53. [6] А. М. Магомедов, Матем. заметки, 85:1 (2009), 65-72. [7] В. Г. Визинг, А. В. Пяткин, Российская конференция “Дискретный анализ и исследование операций” (Новосибирск, 28 июня - 2 июля 2004), Материалы конференции, Изд-во Ин-та математики, Новосибирск, 2004, 6-11. [8] А. М. Магомедов, А. А. Сапоженко, Вестн. Моск. ун-та. Сер. 15. Вычисл. матем., кибернет., 2010, № 1, 39-44.

А. М. Магомедов

Поступило

Дагестанский государственный университет

04.02 .2010

T. А. Магомедов

Дагестанский государственный университет

E-mail: magomedtagir1@yandex.ru 